\title{
Several degenerate vacua and a model for Dark Matter in the pure Standard Model
}

\author{
Holger Bech Nielsen* \\ Niels Bohr Institute, Blegdamsvej 15 -21, Copenhagen \\ E-mail: labechanbi.dr
}

\section{Colin D. Froggatt}

Glasgow University

E-mail: Colin.Froggatteqlasgow.ac.uk

\begin{abstract}
We return to a model of ours for what the dark matter can be with the property that it is compatible with the Standard Model alone. The only genuine new physics is a principle imposing restrictions on the parameters/couplings in the Standard Model. They are required to lead to several vacua being degenerate in energy density with each other. We especially look for some of the signals from the dark matter, which are not just gravitational: The $3.55 \mathrm{keV} \mathrm{X}$-ray radiation, the positron excess and further $\gamma$-ray excess. The picture of ours for dark matter has it being $\mathrm{cm}$-size pearls of 100000 ton mass in order of magnitude.
\end{abstract}

Corfu Summer Institute 2019 "School and Workshops on Elementary Particle Physics and Gravity" (CORFU2019)

31 August - 25 September 2019

Corfu, Greece

\footnotetext{
* Speaker.
} 


\section{Introduction}

\section{Several degenerate vacua and a model for Dark Matter in the pure Standard Model}

We believe in the pure Standard Model further up in energy than most physicists, except for see-saw neutrinos and the baryon number excess for which we accept the need for new physics:

- No new fundamental particles, except see-saw neutrinos and possibly particles almost at the Planck scale; but we shall not talk about such high energies today.

- For fine tuning problems we introduce/propose a new law of nature, that restricts the values of the coupling constants and masses in the Standard Model. In this way we explain, for instance, the hierarchy problem of why the weak scale is so low compared to the Planck scale (or the GUT scale, if it existed) [四, 口, []].

- The new law: There are several vacua/phases of empty space, all having the same energy density/cosmological constant. We call it the "multiple point (criticality) principle" [⿴囗十, [], 目, 团 $=$ MPP.

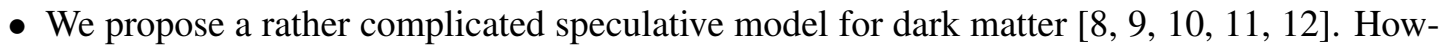
ever it is based on the pure Standard Model only with the help from our fine-tuning law, the "Multiple Point (criticality) Principle" (MPP), using Higgs exchange to bind top and anti-top quarks.

- For neutrino oscillations and the baryon excess we have to accept new physics at the seesaw scale, presumably $10^{12} \mathrm{GeV}$, but no new physics at the LHC except for non-perturbative effects.

- For fine-tuning and the hierarchy problem we make use of our "multiple point principle", which only restricts the couplings and the Higgs mass, but gives no deviation from the pure Standard Model in terms of new particles say.

- Our "Multiple Point (Criticality) Principle" was used before the Higgs particle was observed to predict its mass [133, [4] to be $135 \pm 10 \mathrm{GeV}$

- Hypothesising the existence of three degenerate vacua in the Standard Model (again from MPP), we obtain the right order of magnitude for the weak scale relative to the scale of the second minimum in the Higgs effective potential, which happens(?) to be close to the Planck scale [四, 口, []].

- The Higgs plays a crucial role for our somewhat "complicated" dark-matter speculation.

- About 5 anomalies - seeming deviations from the Standard Model - are explainable in order of magnitude as non-perturbative effects, due to the top Yukawa coupling $g_{t}=0.935$ being "big" [ㄷ5]. 


\section{Dark Matter}

Main points of present talk:

- Our dark matter model/idea of mouse size pearls of a new type of vacuum, to be explained by the effects of Higgs exchange binding top quarks and anti-top quarks.

- Multiple Point Principle: Several vacua with the same energy density separated by high tension "walls" (surfaces much like the surface of a bubble of water).

- Some non-gravitational dark matter signals support our dark matter pearl model:

- The $3.5 \mathrm{keV}$ X-ray radiation [ए], ㅁ], ㅁ]].

- Positrons relative to gamma-rays from dark matter.

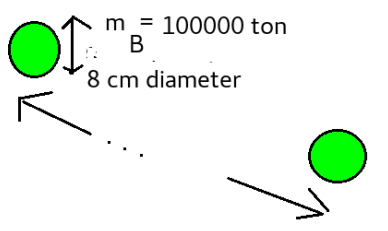

distance as to Moon or Sun

Inside second vacurum with ordi-

nary metter on top.

Our pearls are bubbles of a new vacuum with highly compressed ordinary matter inside

- Our main new physics assumption (which is not really new but should be a non-perturbative effect from the Standard Model): Higgs bosons and top quarks interact so strongly, that a phase of these particles attracting each other had been fine tuned by MPP to get the same energy density as the usual vacuum.

- This gives rise to domain walls separating the two phases with a tension $\sim$ an energy density which by dimensional arguments is given by the top or Higgs masses.

- Our pearls - making up the dark matter by our hypothesis - are bubbles of this new vacuum to which has been added ordinary matter under strong pressure in order to counteract the pressure from the domain wall.

\section{Sharp fall in transparency at $3.5 \mathrm{keV}$}

The pearls will be transparent for radiation of frequency less than the homolumo energy gap $v<3.5 \mathrm{keV}$. However for frequencies $v>3.5 \mathrm{keV}$ the pearls will be highly non-transparent. 


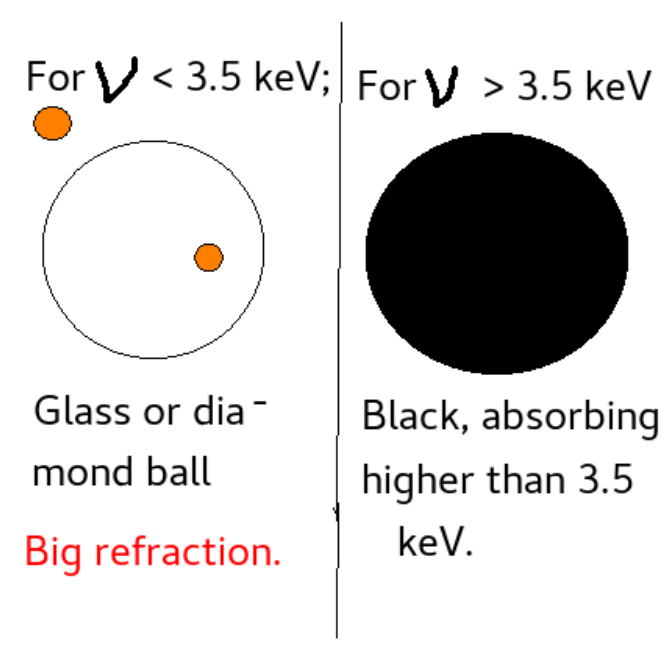

\section{Refracting pearl}

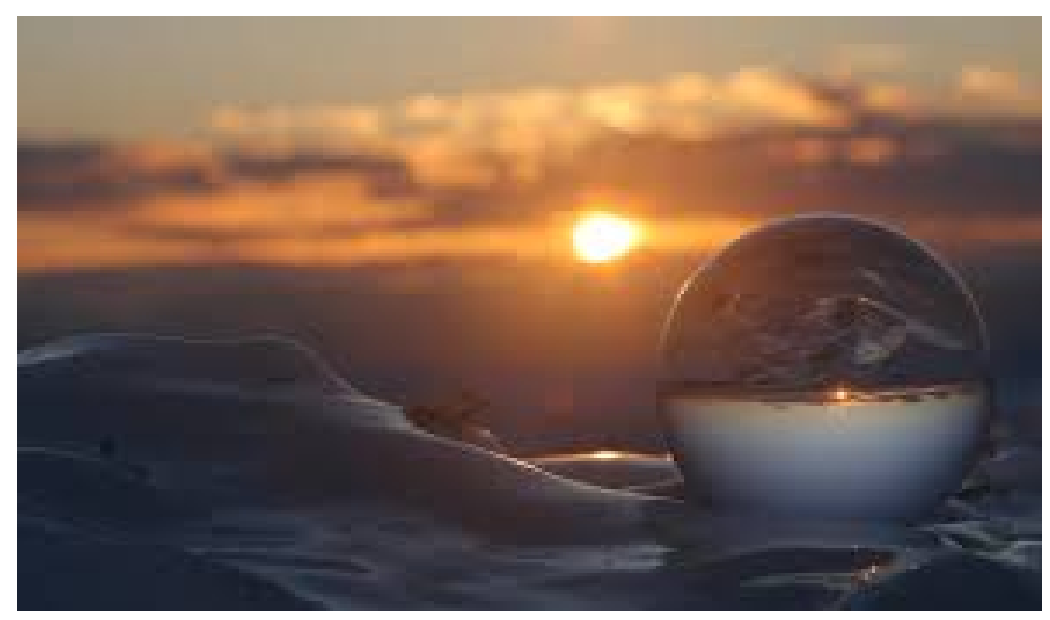

The pearl would have a very high density and therefore also a very high refractive index, which of course is only observable in the frequencies below the homolumo gap energy/frequency $\sim 3.55$ $\mathrm{keV}$. Thus it would make a very deformed and inverted picture of the surroundings, as illustrated in the image above of the sun and the horizon refracted through the pearl.

\section{Homolumo Gap}

We estimate the screening energy per electron in the highly compressed material in the inside of our pearls using the semi-classical Thomas Fermi [ㅍ] approximation. This screening energy functions like a homolumo gap effect [[20], giving a gap in the electron energy spectrum. 


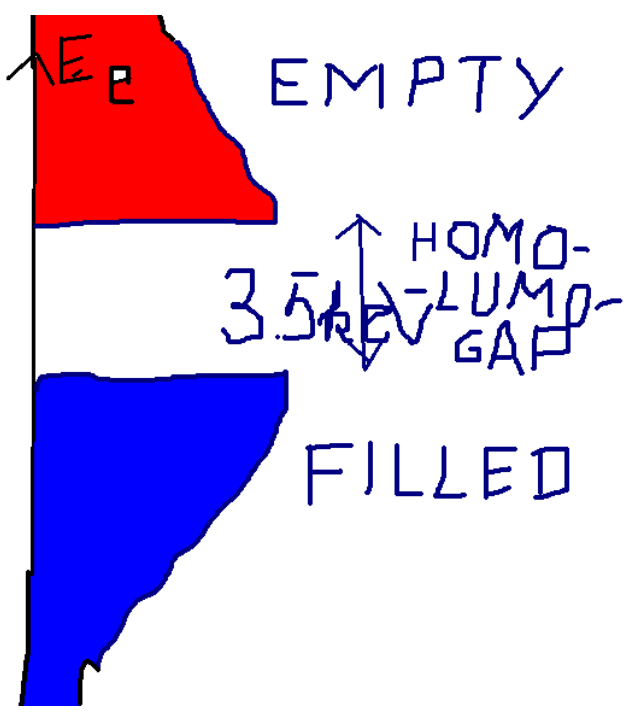

Using this energy gap excitons may be formed as weakly bound states of an electron above the gap and a hole below. Such excitons are now supposed to decay under emission of an X-ray and thus produce the mysterious $3.5 \mathrm{keV}$ line.

\section{Higgs boson exchange binds top and anti-top quarks to make a new vacuum}

- The Higgs field is an even order tensor field like the graviton field and like the latter gives an attraction between "everything" tops and anti-tops.

- The more tops and anti-tops you can put together the stronger they bind.

- But tops are fermions and the Pauli principle limits the number of tops and anti-tops in the same geometrical state to be no more than 12 .

- The 12 top and anti-top ground state is bound so strongly that its mass is estimated to be around $750 \mathrm{GeV}$, similar to that of the now dead "F(750)-digamma" resonance [2], [22].

Diagram giving the binding of 12 top and anti-top quarks to an "F(750)-digamma". 


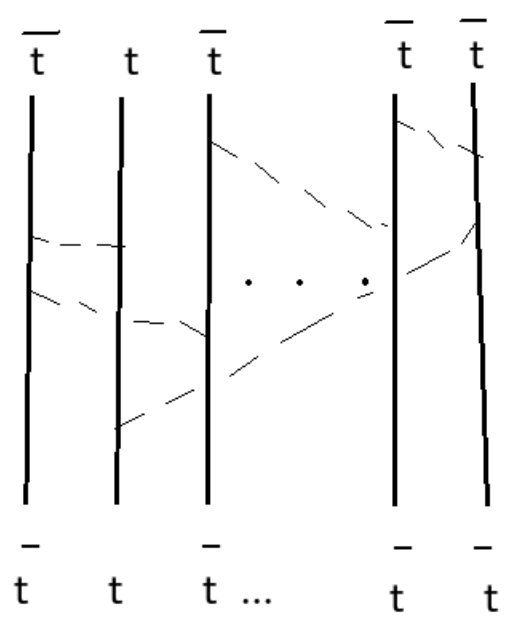

Higgs bosons exchanged between

6 tope and 6 anti-tops.

Inside the pearls: the bound states $F(750)$-digamma plus ordinary matter, e.g. carbon etc.

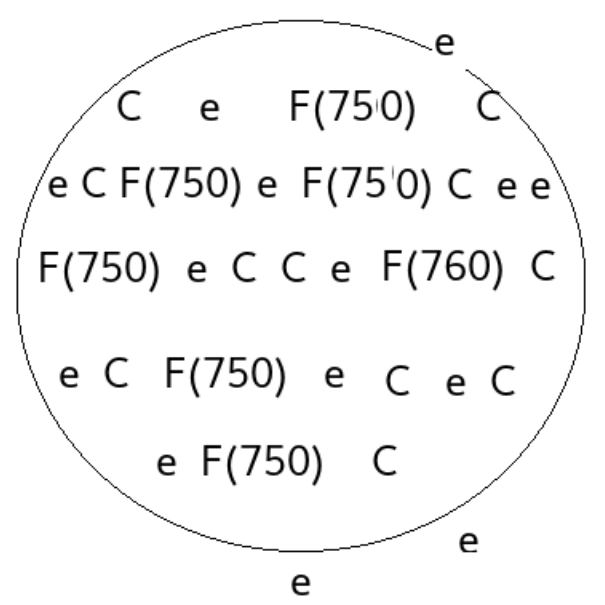

\section{Formation of our Dark Matter Pearls}

At temperatures above the energy scale of the domain walls there are many many walls around. 


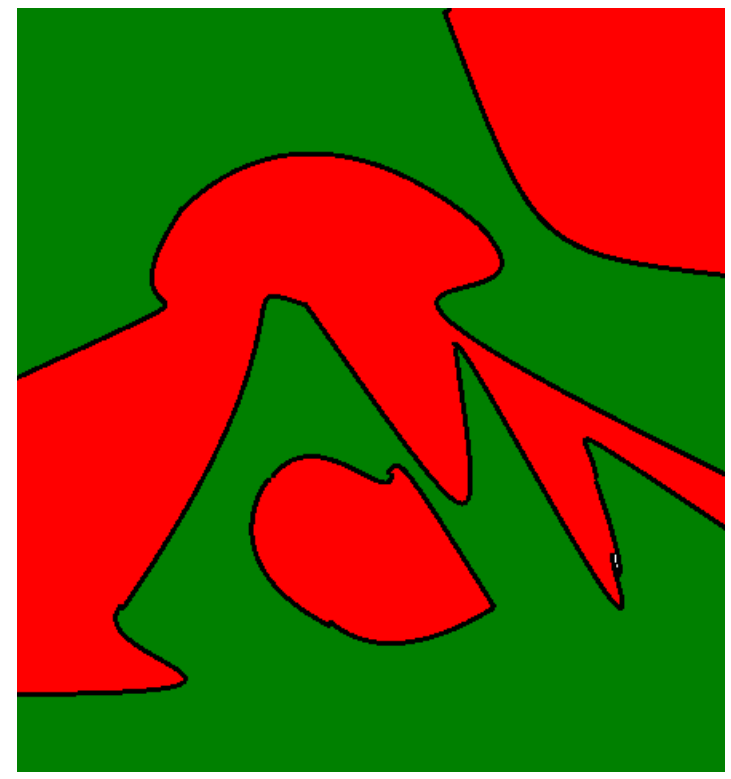

As times goes on, in the early universe, the walls straighten out and one phase may disappear.

- It is crucial that there is no symmetry - such as say a spontaneously broken discrete symmetry - because that causes a catastrophic cosmology.

- With only degeneracy (MPP) there is some difference between the two or more phases (vacua) and one of them will win/take over.

- Only if there is some special mechanism, which can stabilize small pearls of one phase, will a bit of both phases survive. In our case one phase has a smaller Higgs expectation value (probably $\langle\psi\rangle=0$ ) and will attract the baryons say.

- Pearls which are sufficiently large that the pressure is sufficiently low so as not to squeeze its baryons out will be stabilized and survive till today or longer.

Pearls stabilized by their baryon and electron content become very spherical.

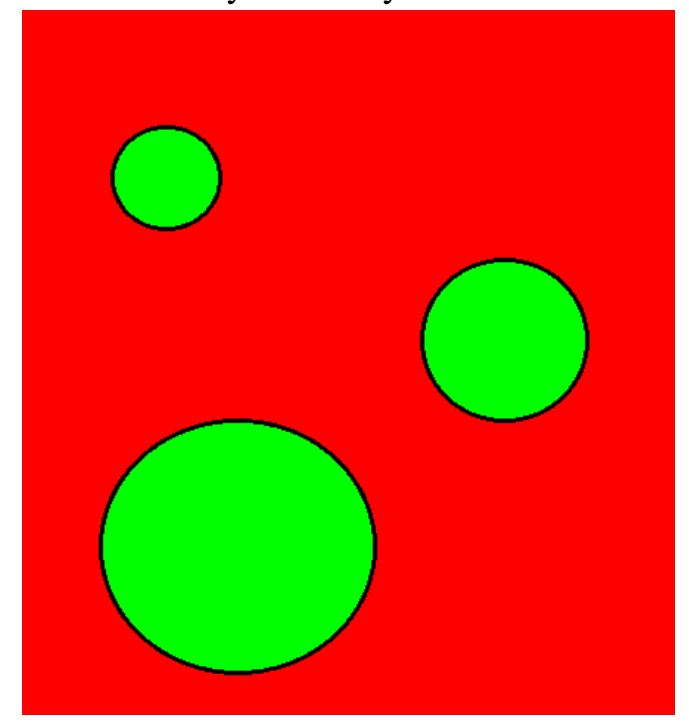


Only sufficiently big pearls survive.

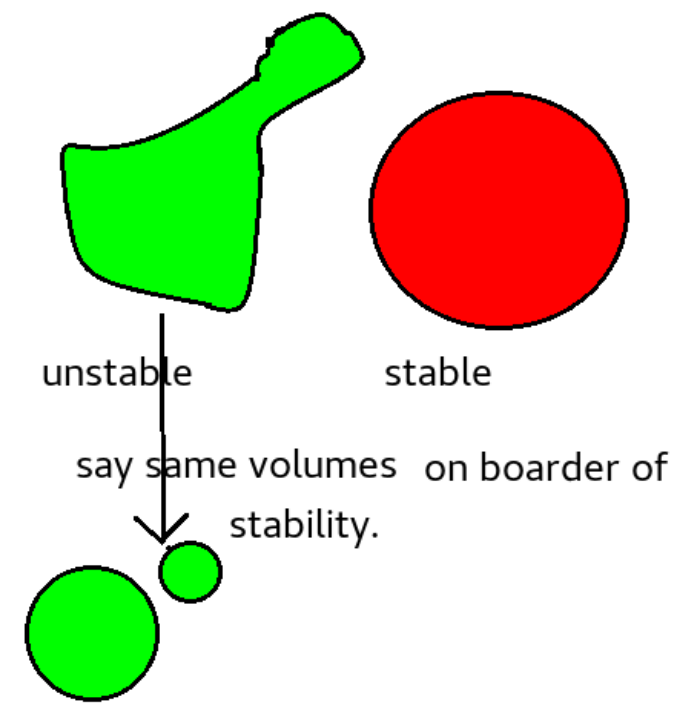

Typically the pearl size is $2^{4 / 9} \sqrt{4 \pi}$ times the formal minimal size needed for stability

- Assuming that the minimal energy $\Delta V$ needed to press a nucleon out of a pearl and the tension in the domain wall around the pearl are known, one can estimate the minimal pearl radius $R_{\min }$ for stability.

- But to avoid breaking up into smaller pieces and thus becoming unstable in the last moment a somewhat bigger size is needed.

- We estimate that typically an extra reserve radius factor $\xi=2^{4 / 9} \sqrt{4 \pi}$ is needed [미], where we define the actual pearl radius to be $R=\xi R_{\min }$.

(In our earlier papers we ignored this factor $\xi$, and only thought about it after seeking to fit the $3.5 \mathrm{keV} \mathrm{X}$-ray radiation intensity.)

\section{5. $3.5 \mathrm{keV}$ X-ray radiation from Dark Matter ?}

Cline and Frey [23] fitted data on the $3.5 \mathrm{keV} X$-ray from Dark Matter from the point of view that the line is due to inelastic scattering of dark matter to an excited state that subsequently decays. The important feature of this model for us is that, as in our model, the intensity of radiation coming from a region of space is proportional to the square of the dark matter density.

\section{Comparing $\frac{N \sigma}{M^{2}}$ to the fit of Cline and Frey}

- In our model the $3.5 \mathrm{keV} \mathrm{X}$-ray radiation is emitted when two of the pearls collide out in the universe, and thus the intensity of the X-rays to be observed must be proportional to the cross section for two pearls colliding $\sigma=\pi(2 R)^{2}$.

- It must of course also be proportional to the number $N$ of $\gamma$ 's with $3.5 \mathrm{keV}$ emitted in such a collision. 
- For a given mass density of of the dark matter, as estimated from its gravity of course, the number density of pearls is proportional to the inverse mass per pearl $1 / M$.

- Since the pearls must meet to collide the rate of $\gamma$-ray emission must be proportional to the square of the matter density and consequently to $1 / M^{2}$.

- Thus the rate to be extracted from the fit to the observations is the combination $\frac{N \sigma}{M^{2}}$.

\section{$3.5 \mathrm{keV}$ Radiation comes after collisions}

\section{$3.5 \mathrm{keV}$ generation}

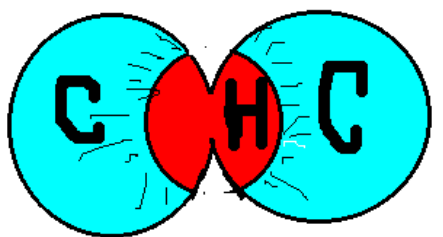

$3.5 \mathrm{keV} X$-ray radia tes from the edge of the hot region through the cold one $C$ out in the outer space arround the two having collided pearls.

Table 1: This table is based on the table 1 of Cine and Frey in reference [23]].

\begin{tabular}{|c|c|c|c|c|c|}
\hline Name & $\begin{array}{c}N<\sigma_{C F} v>* \\
\left(\frac{10 G e V}{M}\right)^{2} \\
10^{-22} \mathrm{~cm}^{3} s^{-1}\end{array}$ & $\mathrm{~km} / \mathrm{s}$ & boost & $\begin{array}{c}\left(\frac{N<\sigma_{C F} v>}{v * \text { boost }}\right) * \\
\left(\frac{10 \mathrm{GeV}}{M}\right)^{2} \\
10^{-27} \mathrm{~cm}^{2}\end{array}$ & Remark \\
\hline Clusters[ए]] & $480 \pm 250$ & 975 & 30 & $0.016 \pm 0.008$ & \\
\hline Perseus[ए[] & $1400-3400$ & 1280 & 30 & $0.037-0.09$ & \\
\hline Perseus[[1]] & $(1-2) * 10^{5}$ & 1280 & 30 & $2.7-5.3$ & ignored \\
\hline Perseus[24] & $2600-4100$ & 1280 & 30 & $0.07-0.11$ & \\
\hline $\mathrm{CCO}$ [प] & $1200-2000$ & 926 & 30 & $0.04-0.07$ & \\
\hline M31[ए]] & $10-30(\mathrm{NFW})$ & 116 & 10 & $0.0086-0.026$ & \\
\hline \multirow{3}{*}{$\mathrm{MW}[25]$} & $30-50$ (Burkert) & & & $0.026-0.043$ & \\
\hline & $0.1-0.7$ (NFW) & 118 & 5 & $0.00017-0,0012$ & ignored \\
\hline & $50-550$ (Burkert) & & & $0.084-0.93$ & in average \\
\hline Average & & & & $0.032 \pm 0.006$ & \\
\hline
\end{tabular}

The notation used in Table 1 is as follows:

- $\mathrm{N}$ is the number of $3.5 \mathrm{keV}$ photons emitted per collision.

- $\sigma_{C F}$ is the cross section as calculated assuming that there is no boost (see below) for the particles of the dark matter to collide. That is to say $\sigma_{C F}$ is calculated as if the density $\rho$ of 
dark matter were given just by the model for dark matter distribution used by Cline and Frey, without any clumping further than the galaxies themselves having been included. The estimated true cross section is thus rather

$$
\sigma=\sigma_{C F} / \text { boost }
$$

- $v$ is the average velocity dispersion.

- boost is the increase in collision rate due to the clumping of the dark matter into sub-halos, which we crudely estimate from references [[26], [27] and [28]. These papers contain computer simulations of gravitational interactions between dark matter constituents in galactic halos forming clumps of much higher density than the average density. Such clumping obviously enhances the true average square density compared to the square of the average density.

- $M$ is the mass of the dark matter particles.

- Since it looks hopeless to get a fit with values for the same quantity differing by a factor 50 or 100, we left out the Boyarsky measurement for the Perseus cluster. It really means that the model used by Cline and Frey with the intensity proportional to the square of the dark matter density is disfavored by the Perseus cluster analysis.

- Because of the uncertainty in the dark matter distribution in the Milky Way center (MW) whether it be NFW or Burkert - we left the Milky Way center out of the averaging; but within uncertainties it is quite consistent with the average.

- The notations "NFW" [2Q] and "Burkert" [B] stand for a couple of different models for the distribution of dark matter in a galaxy mainly deviating by "NFW" having a strong peak at the center of the galaxy.

- MW stands for our Milky Way galactic center.

- M31 is the Andromeda Galaxy.

- CCO stands for a combination of Coma + Centaurus + Ophiuchus clusters.

From Table 1 it follows that the experimental value of $\frac{N \sigma}{M^{2}}$ is

$$
\begin{aligned}
\left(\frac{N \sigma}{M^{2}}\right)_{\exp } & =(0.032 \pm 0.006) * 10^{-27} \mathrm{~cm}^{2} /(10 \mathrm{GeV})^{2} \\
& =(1.0 \pm 0.2) * 10^{23} \mathrm{~cm}^{2} / \mathrm{kg}^{2}
\end{aligned}
$$

It turns out that the quantities whose values we use as tests of our model depend on the single parameter $\frac{\xi * 10 \mathrm{MeV}}{\Delta V}$. These quantities are:

- The frequency of the radiation $3.5 \mathrm{keV}$,

- The intensity related quantity $\frac{N \sigma}{M^{2}}$ of the $3.5 \mathrm{keV}$ radiation,

- The cube root of the surface tension $S^{1 / 3}$ of the pearl, 
- Theoretical values of both $\xi$ and $\Delta V$.

Our calculations are almost just order of magnitude estimates and so we make fits to the logarithms of the above quantities as given in Table 2 .

\begin{tabular}{|c|c|c|c|c|}
\hline Name & $\frac{\xi * 10 \mathrm{MeV}}{\Delta V}$ & $\ln \frac{\xi * 10 \mathrm{MeV}}{\Delta V}$ & Uncertainty & \\
\hline Frequency “3.5keV" & 5.0 & 1.61 & $100 \%$ & \\
Intensity $\frac{N \sigma}{M^{2}}$ & 3.8 & 1.3 & $90 \%$ & \\
$S^{1 / 3}$ theory 1) & 0.28 & -1.3 & $40 \%$ & \\
$S^{1 / 3}$ theory 2) & 1 & 0 & $40 \%$ & \\
Combined theory $\xi, \Delta V$ & 2.4 & 0.88 & $100 \%$ & \\
\hline Ratio $\frac{t_{\text {spread }}}{t_{\text {radiation }}}=1$ & 4 & 1.4 & $80 \%$ & 1. b. \\
\hline
\end{tabular}

Table 2: Table of four theoretical predictions of the parameter $\frac{\xi * 10 \mathrm{MeV}}{\Delta V}$ on which the quantities happen to mainly depend. The first column denotes the quantities for which we can provide a theoretical or experimental value to be expected for our fit to that quantity. The next column gives what these expected values need the parameter combination $\frac{\xi * 10 \mathrm{MeV}}{\Delta V}$ to be. The third column is the natural logarithm of that required value for the ratio $\frac{\xi * 10 \mathrm{MeV}}{\Delta V}$, i.e. $\ln \frac{\xi * 10 \mathrm{MeV}}{\Delta V}$. The fourth column contains crudely estimated uncertainties of the parameter thus fitted counted in this natural logarithm. In the last column we just marked the ratio $\frac{t_{\text {spread }}}{t_{\text {radiation }}}$ with l.b. to stress that it is only a lower bound and shall not be considered a great agreement for our theory. We refer to our paper [ㅁ] for a discussion of this ratio and of our two theories for the surface tension $S$.

Using our theory 2) for the surface tension we obtained the following fit:

$$
\begin{aligned}
<\ln \frac{\xi * 10 \mathrm{MeV}}{\Delta V}> & =0.57 \pm 0.3 \\
\Rightarrow<\frac{\xi * 10 \mathrm{MeV}}{\Delta V}> & =\exp (0.57)=1.8_{-0.5}^{+0.6} \\
\chi^{2} & =4.95 \text { (for } 4 \text { degrees of freedom) }
\end{aligned}
$$

This our best fit including everything gives the following overall fitted values for the experimental quantities

$$
\text { The frequency predicted " }\left.3.5 \mathrm{keV}^{\prime \prime}\right|_{\text {theory }}=10_{-7}^{+19} \mathrm{keV} \text {, }
$$

and

$$
\text { The intensity predicted }\left.\frac{N \sigma}{M^{2}}\right|_{\text {theory }}=4.7_{-2.8}^{+6.9} * 10^{22} \mathrm{~cm}^{2} / \mathrm{kg}^{2}
$$

which is to be compared with

$$
\left.\frac{N \sigma}{M^{2}}\right|_{\exp }=(10 \pm 2) * 10^{22} \mathrm{~cm}^{2} / \mathrm{kg}^{2}
$$




\section{Other heating of Pearls}

- Very strangely: If $3.5 \mathrm{keV}$ radiation should come from big clusters of dark matter, it should NOT come from a supernova-remnant; but it was seen from the Thyco Brahe's supernova remnant [B]]!

In our model this may be explained by the fact that the cosmic radiation from the remnant of the supernova provides our pearls with energy. The pearls then radiate that energy out as characteristic radiation of frequency $3.5 \mathrm{keV}$, due to the excitons formed in their interior.

\section{Systematics?}

Tycho Supernova Remnant

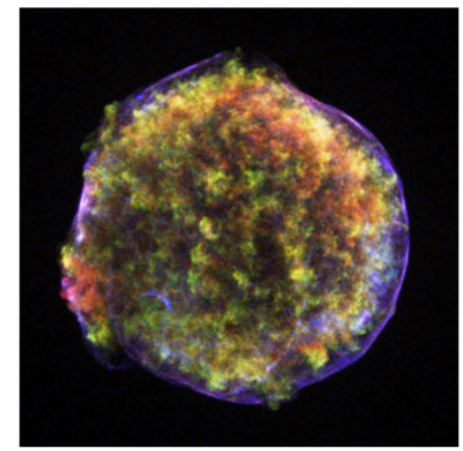

175 ksec XMM observations

Line at $3.55 \mathrm{keV}$ detected:

$>$ potassium with high abundance?

$>$ systematics in line flux?

$>$ NOT dark matter

Credit: NASA/CXC/Rutgers/Warren, Hughes et al.

Jeltema \& Profumo 2015

\section{Positrons from dark Matter ?}

One of the hopes for seeing something other than gravitational effects from dark matter is the observation that above $9 \mathrm{GeV}$ or so the ratio of the number of positrons to the combined number of electrons plus positrons in cosmic radiation goes up with energy. This is unexpected as the ratio should fall if the standard secondary production of positrons during the propagation of cosmic rays in the galaxy is alone responsible for the positrons.

The speculation now could be that the dark matter could itself produce these excess positrons either by decay or by annihilation or, in our model of dark matter, via collisions of the dark matter pearls with each other.

To estimate the absolute amount of positrons needed to be explained as coming from the dark matter let us first, for normalization purposes, remark that in the energy range per positron or electron around $10 \mathrm{GeV}$ the flux of positrons or electrons counted together is of the order

$$
\operatorname{flux}\left(e^{+}+e^{-}\right)=150 E^{-3} m^{-2} s^{-} \text {ster }^{-1} G_{e V}
$$


per $\mathrm{GeV}$ in the spectrum.

Taking $20 \mathrm{GeV}$ as a typical energy of the positron we have, according to the PAMELA observation [32], the ratio of positrons to the sum of both positrons and electrons is about 0.05 , while the expected value with only the "normal" production mechanism would rather be 0.03 . As one looks higher up in energy towards $100 \mathrm{GeV}$ where the data stop, the positrons get relatively more and more copious. But of course on the background of an energy $E$ to the third power fall off, it means that the positron rate falls off a bit slower than the one of the electrons.

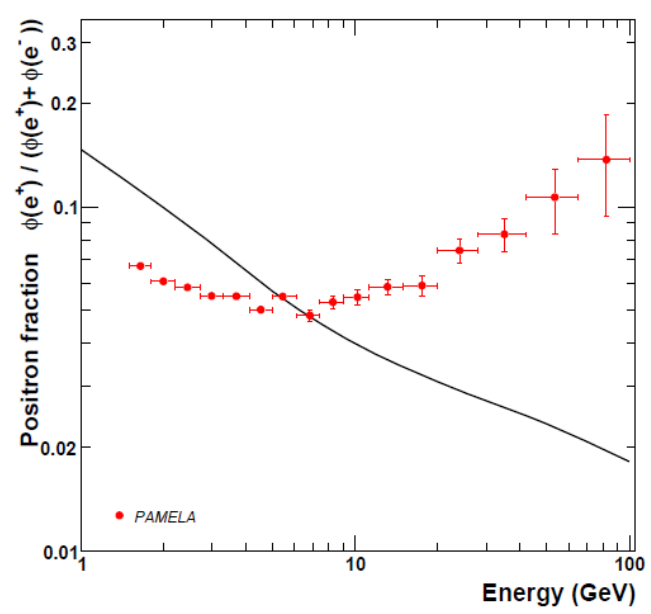

Figure 1: In the Pamela experiment [B2] it is found that the ratio of positrons to (electrons plus positrons) in cosmic rays does not follow the theoretical expectation, which is here shown by the continuous curve [B33]. The data seem to increase with the energy of the positron and become bigger than the expectation from about $9 \mathrm{GeV}$. The idea is that this excess above $9 \mathrm{GeV}$ could be due to dark matter.

Taking very crudely the result at $20 \mathrm{GeV}$ that there is an (absolute) excess of positrons of (0.05- 0.03)*150 $\mathrm{E}^{-3} \mathrm{~m}^{-2} \mathrm{~s}^{-1} \mathrm{ster}^{-1} \mathrm{GeV}^{2}$, we may imagine integrating up over the spectrum in the neighborhood of the energy $20 \mathrm{GeV}$ or $10 \mathrm{GeV}$ to obtain for the order of magnitude of a flux of positrons:

$$
\begin{aligned}
\int_{\text {region } \sim 20 \mathrm{GeV}} \operatorname{flux}\left(\mathrm{e}^{+}\right) & \approx 150 * 1 / 20^{2} * 0.02 \mathrm{~m}^{-2} \mathrm{~s}^{-1} \text { ster }^{-1} \\
\text { giving an energy flux } & \\
\text { "energy flux" } & \approx 150 * 1 / 20 * 0.02 \mathrm{GeVm}^{-2} \mathrm{~s}^{-1} \text { ster }^{-1} \\
\text { meaning "energy density" } & \approx 150 * 1 / 20 * 0.02 /\left(3 * 10^{8}\right) * 4 \pi \mathrm{GeV} / \mathrm{m}^{3} \\
& \approx 0.6 * 10^{-8} \mathrm{GeVm} \mathrm{m}^{-3} \\
& =0.6 * 10^{-14} \mathrm{GeV} / \mathrm{cm}^{3} .
\end{aligned}
$$

The dark matter density in our solar neighborhood is $0.3 \mathrm{GeV} / \mathrm{cm}^{3}$. This means that very crudely around us the suspected excess of positrons has an energy density about a factor $10^{14}$ smaller than the energy density of the dark matter from which we suspect it to come.

When rather seldomly two of our dark matter pearls collide with each other and unite their common skin is contracted to form the smaller surface of bubble skin around the two united pearls. 
We imagine that part of the energy released by the skin contraction is transformed into making and accelerating positrons as well as to accelerate mainly electrons. We must now estimate whether the positrons produced and accelerated in this way, and having not yet disappeared by losing energy or annihilating with electrons around, could have an energy of the order of $10^{-14}$ times the Einstein rest mass energy of the dark matter from which it comes.

\section{Positrons and $\gamma$-rays from Dark matter.}

- When two of our dark matter pearls collide and the skin/domain wall around them contracts with high surface tension $S$, a region is heated to about $\mathrm{MeV}$ temperatures and large amounts of the degenerate electrons in the pearl are spit out.

- Some of the electrons may run out to a long distance from the two united pearls and they will create an electric field able to accelerate positrons (which are probably also being produced in some amount) at first estimate up to a few $\mathrm{MeV}$ energies, but a few will get much more.

- In any model with positrons being produced and accelerated, such positrons will shake off $\gamma$-rays/light.

- The emission of such light has an intensity proportional to the square of the acceleration of the charged particle, here the positron.

- In "usual" dark matter models - a particle decaying and producing the positron - the positron is accelerated over a very short distance, effectively given by the mass of the particle and quantum mechanics.

- In our model the acceleration takes place over a distance given by the extent of the electron cloud from the explosion. At least it is "macroscopic".

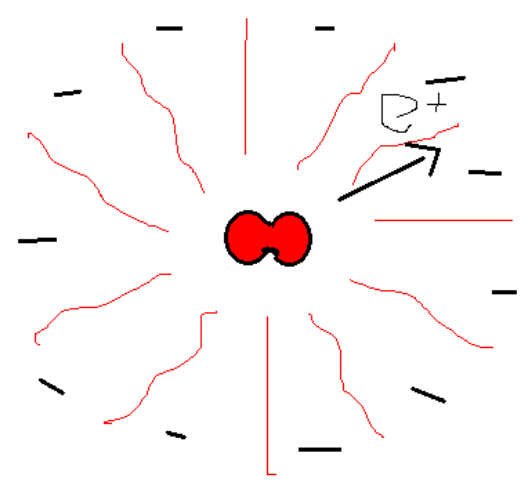

Positrons accelerat ed over long

distance relative to positrons from

a decay.

Figure 2: On the figure we seek to illustrate that the electrons have been slung far away and produced an electric field that potentially could drive e.g. the positrons. 


\section{Time needed for positron production}

In the simple model of a sterile neutrino of mass two times $3.5 \mathrm{keV}$ decaying into a photon plus a normal neutrino, one fits the life-time of the sterile neutrino supposed to be the dark matter particle to be $\tau_{D M} \sim 10^{27.8 \pm 0.3} s$ [B4]. This means that half the (Einstein) energy of the dark matter would go into the $3.5 \mathrm{keV} \mathrm{X}$-ray line with a decay rate given as the inverse of this life-time. Now when our pearls collide and produce a lot of $3.5 \mathrm{keV} \mathrm{X}$-rays from the energy released, according to our model only of the order of $0.4 \%$ of the (Einstein) energy of these dark matter pearls actually goes into the X-rays. So counted in terms of how much of the energy of the dark matter decays into X-ray energy in the $3.5 \mathrm{keV}$ line, our model is less effective - having a smaller rate - than the sterile neutrino model by a factor of the order $0.4 \% /(1 / 2) \approx 1 / 100$. So, thinking in terms of the mass or energy decaying, the effective life-time in our model is about one hundred times longer than that for the sterile neutrino model mentioned above $10^{27.8} s=0.63 * 10^{28} s$, i.e. an effective life-time $0.63 * 10^{30} s$. Thus the time needed to produce $10^{-14}$ times as much energy in positrons as the energy of the dark matter itself is

$$
t_{\text {needed }}=10^{-14} * 0.63 * 10^{30} s=6.3 * 10^{15} s
$$

Luckily for our model, this time $t_{\text {needed }}=6.3 * 10^{15} \mathrm{~s}$ needed for the positrons to come from the dark matter is 70 times smaller than the age of the universe, which is 13.7 milliard years $=4.3 * 10^{17} \mathrm{~s}$.

But this means the survival time for positrons having been produced by the collisions of the dark matter should be at the very least $1 / 70$ of the age of the universe. If not all the energy from the collisions should go into positrons, it should be even longer!

Positron Excess could be realized in Our Model for Dark Matter.

- If the "fraction of energy going to positrons in the collisions" times "the fraction of the universe age the positrons can keep on running around in the neighborhood" can be bigger than $\sim 1 / 70$, then the observed positron excess could match our dark matter model.

- The usual dark matter decay or annihilation models of the positron excess suffer from the problem of predicting a too large accompanying $\gamma$-ray flux. It may be quite complicated and model dependent to obtain our $\gamma$-ray prediction relative to the positron production. However the positrons in our model are accelerated over much longer - basically macroscopic distances than in simple WIMPs or the like decaying or annihilating type of models. So we get a much smaller acceleration squared for the positrons and thus a much smaller $\gamma$-ray flux than in the "usual" type of model.

\section{Multiple Point Principle (our only new physics)}

\section{Propaganda for "Multiple Point (Criticality) Principle"(=MPP).}

This "multiple point (criticality) principle" is really a new law of nature, which we want to propose and argue for in different ways. The law says that "there are several different vacua, and all these vacua have very small energy densities (or in the older version: the same energy density)". These "vacua" are to be thought of as phases of space, much like that space filled with water molecules, say, can be in a phase of fluid water, water vapor or ice. When we talk about vacua 
instead of just phases we mean that we think of phases which are Lorentz invariant, in the sense that if you imagine moving inside such a vacuum you cannot determine in any way your velocity relative to the vacuum, without observing something else. There is so to speak a relativity principle, like in the theory of relativity, such that the velocity of one of the vacua makes no sense. This of course is contrary to if you were moving through a phase of water; in that case it makes perfect sense to ask for the velocity relative to the water. So the phase of e.g. fluid water is not what we call a vacuum, but if it had been completely impossible to define a velocity relative to it we would have called it a vacuum.

So our postulated new law of nature states that whenever you find such a phase with a valid relativity principle on it, so that it can be considered a vacuum, then this phase shall have the same energy density as all the other phases of this relativistic invariant type, deserving therefore to be called vacua.

As just stated we talked about two versions: the old version in which the statement of the law of nature is only that the different Lorentz invariant phases, vacua, have the same energy densities, and a - we can call - newer version, for which we thank Leonard Susskind for a private communication, that says that all vacua have very small or zero energy density. (The advantage of this latter version is that then you can consider the smallness of the cosmological constant - a great mystery - as a special case of our new version of the multiple point principle.)

There are actually several points which encourage us to believe that nature indeed satisfies this law of nature, the multiple point principle:

- PREdiction : We - CDF and HBN - PREdicted the mass of the Higgs boson from MPP, before it was discovered [123, [4], 40]].

In figure [ 3 one even sees that the value for the Higgs mass was painted long before the Higgs particle was found.

- Phenomenology: In some models we have success phenomenologically: E.g. PREdicted the number of families [ [6, [35, [36]. The predicted top-Yukawa coupling $g_{t}=1.00 \pm 0.14$ agrees with the observed value $g_{t}=0.935$ [ㅈ]]. Scale problem: Why is the Higgs mass $<<$ Planck scale, say? [U, [1], []]

- Theory Theoretical predictions are made in a global time perspective

By this rather cryptic statement of "global time perspective" is meant that we do not assume the usual locality, but that at least coupling constants can feel even the future by being in our typical model say functions of integrals over all space-time of some field combinations [四, [1, 口, [4], [37].

Thinking of the multiple point principle as concerning just some special phases, one may be encouraged by the analogous situation that one actually sees quite often - especially in e.g. Denmark and Scotland - what in English is called slush, namely that you see both ice and fluid water at the same time. Then, by seeing that, you in fact know that the temperature is just $0^{0}$ Celsius. It is namely only at this temperature that there can be a balance in pressure and temperature between both ice and fluid water at the usual pressure of one atmosphere. From the coexistence of the two phases we can so to speak extract knowledge of one parameter, as here said the temperature. 


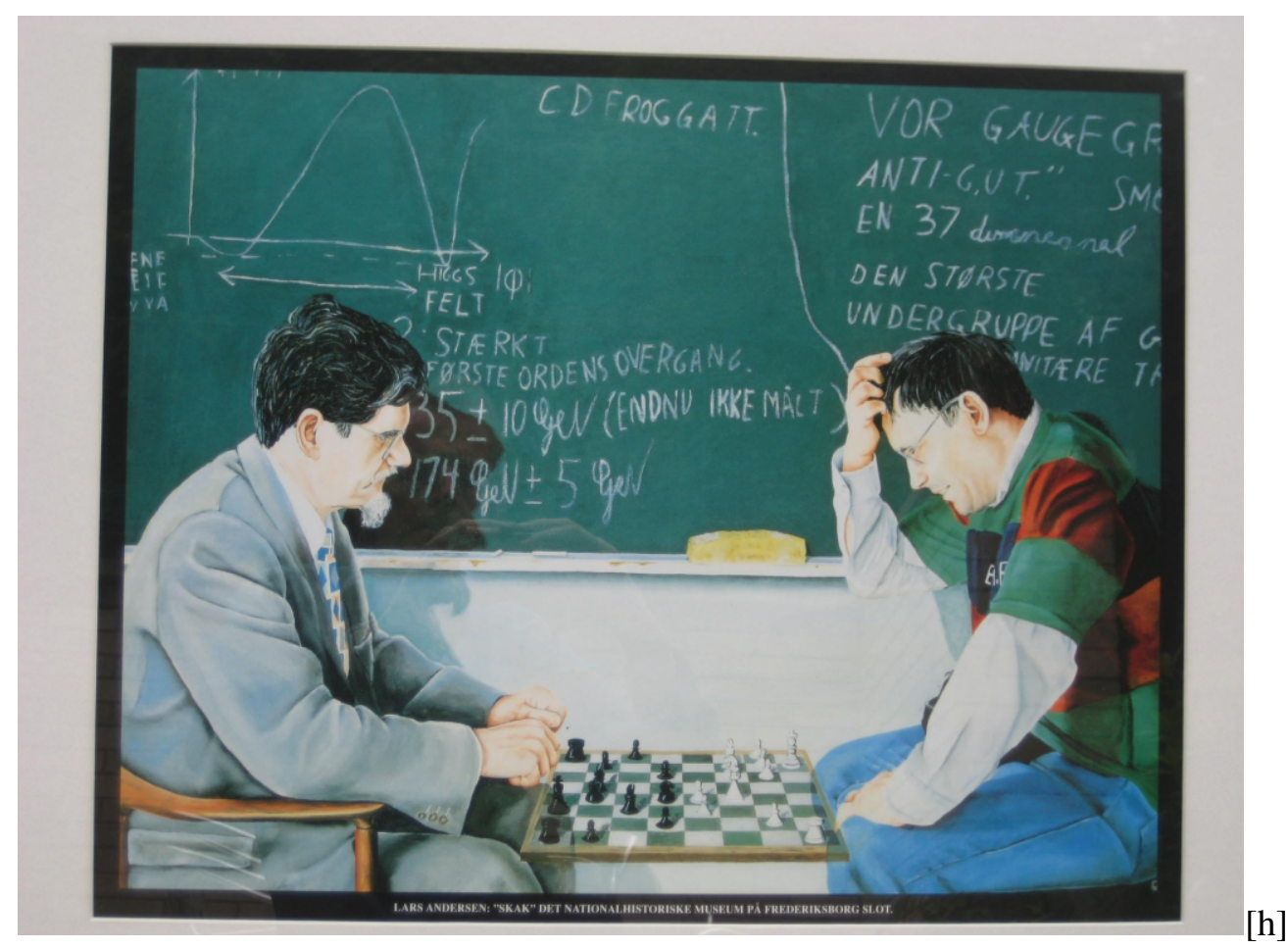

Figure 3: This painting by Lars Andersen, belonging to Frederiksborg Museet, shows that one of us (HBN) was even painted and the painting exhibited long before(Dansk Kunst 1998) the Higgs particle was found in CERN in 2012, so that nobody could at that time know the mass. Nevertheless the mass $(135 \pm 10)$ $\mathrm{GeV}$ is visible except for the 1 behind the head of the previous member of the cabinet in Denmark Mogens Lykketoft. Notice the name of another one of us C. D. Froggatt on the top of the painting. We note that in our paper [[1]] we assumed the Higgs field expectation value in the second vacuum to be equal to the Planck energy and predicted the then poorly known top quark mass to be $(174 \pm 5) \mathrm{GeV}$, which is also shown in the painting. Had we instead used the top quark mass as input, we would still have predicted the Higgs mass and obtained a Higgs vacuum expectation value of the order of the Planck scale.

The main application of the multiple point principle is precisely of this type also: by assuming the coexistence of different vacua (phases) with the same coupling constant and mass parameters, we can deduce a relation between these couplings and mass parameters.

This was what we did when we PREdicted the Higgs mass: We postulated that a vacuum phase (i.e. a relativistic invariant phase) with a very high Higgs field (close to the Planck scale of energy) was degenerate (had the same energy density and thus could coexist in a rather stable way) with the present vacuum surrounding us. Then, considering all the other parameters in the Standard Model as already given, we took the Higgs mass to be adjusted to the degeneracy. This turned out being the right value later on for this Higgs mass. With today's knowledge [B8] we would have predicted $129.4 \pm 1.8 \mathrm{GeV}$ for the Higgs mass to be compared with the actually found one $125.38 \pm 0.14$ $\mathrm{GeV}[\mathrm{BQ}]$.

Since our principle is so similar to requiring the coexistence of different phases, as is what happens in the situation of the existence of slush, you could be tempted to even call our principle a "slush-principle". 


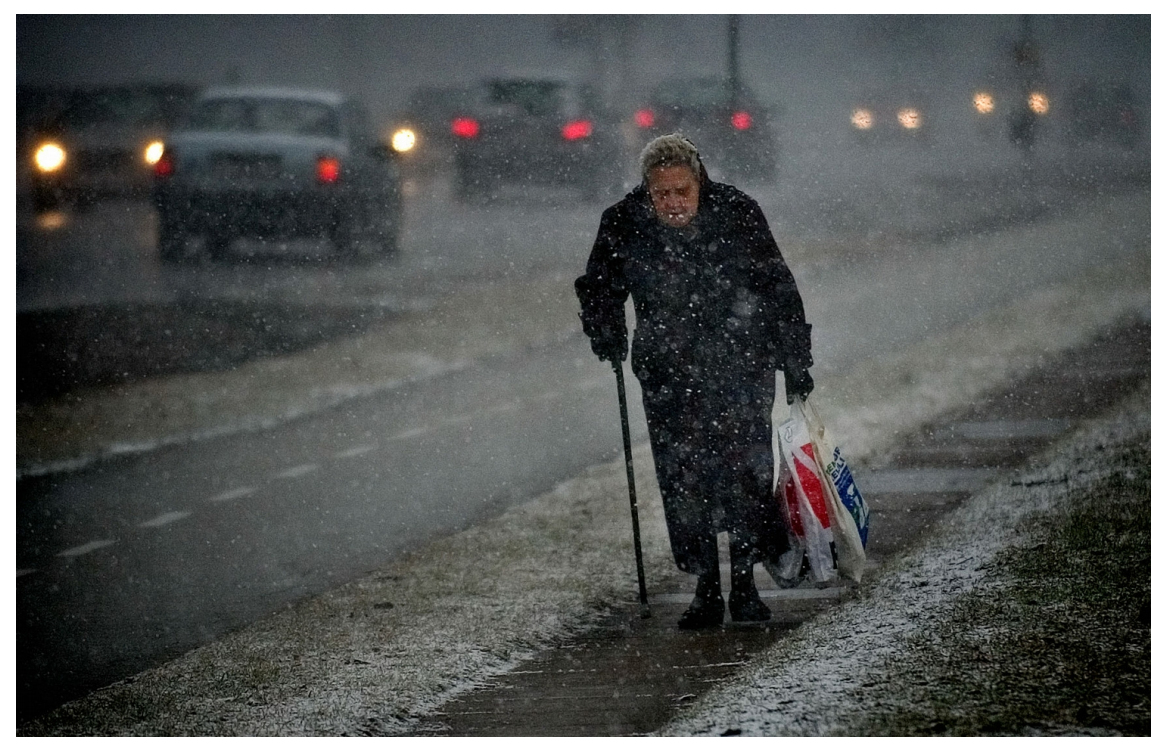

Figure 4: This picture should illustrate that just by looking at it and seeing slush (both ice and fluid water) the reader should be so experienced that he intuitively - by experience - feels how cold it must be and thus e.g. what clothes the people in the picture should wear. This is namely the main point for the multiple point principle: ice and water together give a special temperature $0^{0}$ (when the pressure is one atmosphere).

Usually we think that, since the coupling constants and the other parameters in the Lagrangian density have been the same forever, they had the same values even far back in time shortly after the big bang when a piece of relatively empty space, with a cosmological constant of the order found astronomically today, would hardly be of any significance. But if so it is hard to see how the sameness or smallness of energy densities of the order of cosmological constants at that time could have had any significance. But if so, then the coupling constants and mass parameters in the Lagrangian density could only have taken on their multiple point principle adjusted values provided they got them via a mechanism which was precognizant, i.e. which was influenced by the future in some way.

We therefore tend to think that in order to make a theoretical "derivation" in some way of our new law of nature, the multiple point principle, one should at the outset take a model in which there is not full causality. Then at least coupling constants and mass parameters can be influenced from what goes on in the future. We have worked on some such very speculative models and, once you accept there can be influence from future, it is much easier to derive the multiple point principle.

However, it seems that Gia Dvali has found a derivation of the multiple point principle, in which it appears that he did not assume any breaking of the usual principles of locality. His main point is that Lorentz invariance would enforce some divergent terms, which could only be avoided when the different vacua have the same energy densities.

The abstract of Dvali's paper [4] "Safety of Minkowski Vacuum" states: We give a simple argument suggesting that in a consistent quantum field theory tunneling from a Minkowski vacuum to a lower energy vacuum must be impossible. Theories that allow for such a tunneling also allow for localized states of negative mass, and therefore, should be inconsistent.

Fixing Extensive Quantities (say energy) 
The "derivation" of our new law, the multiple point principle, which we would now like to give is based on the crucial assumption that somehow Nature settles on extensive quantities rather than on intensive quantities to be given a priori.

We know these different possibilities from chemistry or thermodynamics: One can put some specified amounts of various types of molecules and a specified amount of energy into a bottle of a given size. Quantities like the amount of molecules (measured in mol) of a substance or the energy are called extensive, contrary to quantities like the temperature or pressure, or chemical potentials which are called intensive.

The extensive quantities are integrals over the whole sample, while the intensive quantities are locally observable.

Our analogy to this distinction between intensive and extensive quantities deviates a little bit from the usual chemical one by seeking to make our discussion four dimensional so that e.g. our analogues to the extensive quantities are integrals over both space and time of Lagrange-like field expressions $\mathscr{L}_{i}(x)$,

$$
\begin{aligned}
\int \mathscr{L}_{1}(x) d^{4} x & =\text { fixed }_{1} \\
\int \mathscr{L}_{2}(x) d^{4} x & =\text { fixed }_{2} \\
& \cdot \\
\int \mathscr{L}_{N}(x) d^{4} x & =\text { fixed }_{N}
\end{aligned}
$$

If nature specifies these four-dimensional integrals, instead of specifying first the coupling constants and mass parameters in the Lagrangian, then the couplings and mass parameters must be adjusted by nature to give the pre-specified extensive integrals over four-dimensional space-time. But then our hope is that achieving this adjustment turns out to require there to be several phases, several vacua say. This is analogous to the situation in a microcanonical ensemble where, when you specify the energy in a sample, very often you cannot achieve a solution unless two phases are present. This situation where two phases are in equilibrium corresponds to a phase transition point in say temperature (if we fixed the energy of the whole sample) and this is where the slush comes in. If slush is present, the temperature must be zero.

So the hope in our model is that several phases - several vacua - may be needed and they have to coexist. This coexistence means that they should be realized in the same physics, meaning with the same coupling constants and mass parameters in the Lagrangian density. The bare coupling constants should so to speak be the same all over the space-time.

This kind of mathematical problem of determining relations between the coupling constants and mass parameters from the specification of the extensive integrals is very much analogous to the determination of the pressure and temperature in a bottle with pre-specified volume, number of mol of water molecules and energy. This specification can lead, in a range of values for these extensive quantities which is not of null measure, to the requirement that the temperature and the pressure must be at the critical point! That is the point of coexistence of all the three phases fluid water, ice and vapor. The idea is that - supposing we know the form of the Standard Model or some other model - we can calculate the analogue of such a critical point (possibly with even more than three phases meeting, so that it should be called "multi-critical") and then that is our multiple point 
principle prediction. That is what we claim to have had some success with. Really we expect the coexisting vacuum-phases to have the same energy densities, as stated in the above formulation of the principle.

\section{The Problem of why the Weak Interaction Energy Scale is so Small Compared to Say the Planck Scale}

One of the great mysteries or fine tuning problems is the question of why the weak energy scale is so low by a factor of about $10^{17}$ than the supposedly fundamental scale given by the Planck energy scale of $10^{19} \mathrm{GeV}$. Even if one does not accept the Planck scale as the fundamental one, but use instead e.g. grand unification models one tends to get scales of energy much higher than the weak energy scale of only of the order of $100 \mathrm{GeV}$. In this light it becomes a mystery how the Higgs mass-parameter, which determines the weak scale, ever got to be so low. It is of course connected with the fact that the renormalized (dressed) Higgs mass is very small compared to the Planck scale or unified scale. So it is a fine-tuning problem: Why is this renormalized Higgs mass fine-tuned to be so small compared to the Planck mass, say?

Often one discusses the hierarchy problem in which one then accepts, as being more admissible, the fact that the bare Higgs mass squared was tuned in to be very small or even maybe zero. But then one has the hierarchy problem, which makes it even more clear that something is not understood, wrong or rotten: The calculation of the correction to get from the bare Higgs mass squared to the renormalized Higgs mass squared is divergent or, if you renormalize to e.g. some GUT-scale, very big. So it now becomes the mystery of how the bare mass can adjust itself in such a funny way that it just compensates the huge corrections to produce, in the sum, a very small renormalized Higgs mass squared? For this problem it has been claimed that supersymmetry can help, because it converts the corrections, which a priori are quadratic, into logarithmic corrections and therefore much smaller.

But SUSY particles have yet to be found and supersymmetry must be broken. So the correction that cannot be suppressed by supersymmety gets bigger as experiments continue to exclude SUSY. We must already put the SUSY breaking scale so high that it is a bit accidental, a bit of fine-tuning may even be required one could say, then to get such a small Higgs mass as one finds.

Using the multiple point principle we take a somewhat different point of view: Supersymmetry cannot leave us without fine-tuning, even if we took the assumption of a small bare mass squared as acceptable. So there is no way except by accepting some fine-tuning! Now the multiple point principle comes in as a fine-tuning law! We can look at the coupling constant restrictions from the multiple point principle as a kind of fine-tuning.

This way of looking at our principle as a law of fine-tuning opens up the possibility that it can help to solve some of the fine-tuning problems in physics.

Indeed it turns out that the scaling problem we mentioned above can be solved by the multiple point principle, in the sense that our principle can explain why the weak scale is so extremely low compared to the Planck scale or some other fundamental scale. Actually it is the scale of the vacuum with a high Higgs field that comes in. This high Higgs field scale, accidentally you may say, turns out from measured values of the gauge coupling constants to be approximately the Planck scale. In any case it is a very high scale, essentially the Planck scale. 
Now our main point is: Requiring the existence of three degenerate vacua imposes such restrictions on the coupling constants that indeed the renormalized Higgs mass squared becomes "exponentially small" compared to the scale of the vacuum with the high Higgs field of the order of the Planck scale.

Let us explain this in a bit more detail. We assume that in the pure Standard Model there are three vacua, which then by our multiple point principle must have their energy densities fine-tuned to be very small, essentially zero.

We already talked about these three vacua:

- 1) The vacuum in which we live called the "present vacuum".

- 2) The vacuum inside the dark matter pearls, called the "condensate vacuum", because we speculate that this vacuum has a boson condensate of some bound state of 6 top and 6 anti-top floating in it.

- 3) The vacuum with the high Higgs field expectation value being of the order of the Planck scale. (It was by using the requirement of the same energy density for this vacuum and the present one, that we derived the Higgs mass prior to the Higgs discovery.)

Now the energy density for high Higgs fields - the effective potential for the Higgs field for high Higgs values - is dominated by the Higgs self interaction term in the energy density. So it is a good approximation to calculate the effective potential for a high Higgs field by just putting in the renormalization group running value of this self coupling $\lambda_{\text {run }}(\phi)$ taken at the scale given by the Higgs field $\phi$. In order to have a vacuum at high Higgs field with a small energy density, we require this running self coupling to be very small at that scale and to have a minimum there. Considering the fine-structure constants $\alpha_{1}(\phi)$ and $\alpha_{2}(\phi)$ for the electro-weak gauge fields as given, the presence of such a needed minimum implies that the top Yukawa coupling $g_{\text {t run }}(\phi)=0.4$ at the high Higgs field vacuum scale, where the Higgs field $\phi$ is of the order of the Planck scale.

On the other hand:

Our bound state of the 6 top +6 anti-top quarks is bound by Higgs exchange between the constituent top quarks. Thus the physics of the "condensate vacuum" needing this bound state requires that the Higgs is not heavier than this scale. The Higgs mass scale is essentially what we call the weak scale, since the $W^{ \pm}$and $Z^{0}$ get their masses from the Higgs expectation value. Therefore the weak scale must be at least lower than or the same as the scale where the top and anti-top quarks bind so strongly that the vacuum inside the dark matter pearls can be achieved. But now to have such a strong binding as to produce the condensate phase we calculated in ref. [R]], including a lot of corrections, that a running top-Yukawa coupling $g_{t}$ run $($ condensate scale $)=$ $1.00 \pm 0.14$ was needed.

The above use of the multiple point principle twice gives rise to the two important requirements:

- At the scale of the high Higgs field vacuum (essentially the Planck scale) one needs that the running top-Yukawa coupling is $g_{\text {trun }}(\sim$ Plank scale $)=0.4$. 
- At the scale of "condensate vacuum", which we take to be higher than or equal to the weak scale, this same running coupling should be of order $g_{t}$ run $($ condensate scale $)=1.0$, in order that the binding be sufficient to have the "condensate vacuum" at all.

These two requirements, both coming from the multiple point principle, obviously call for a significant running of the top-Yukawa coupling $g_{t \text { run }}(\mu)$ over the scale range between the high Higgs field vacuum scale and the scale connected to the "condensate vacuum", which must at least be as high as the weak scale.

But now the couplings going into the $\beta$-functions describing the running are of the order of the electroweak fine structure constants and actually give rise to beta functions which are rather small. (One could say that the smallness of the coupling constants compared to the values which would give rise to beta functions of order unity is a small fine-tuning problem; but it is very small in magnitude compared to the scale problem we discuss here). So we have a relatively slow running and need the Yukawa coupling $g_{t}$ run $(\mu)$ to "run" from 0.4 to 1.0. This takes then relatively many orders of magnitude in energy scale. In fact a numerical estimate gets order of magnitude-wise very close to post-dicting the scale of the "condensate vacuum" to be very near to what we know the weak scale is!

So we even obtained an approximate numerically correct prediction for the logarithm of the weak scale of energy from the multiple point principle theory.

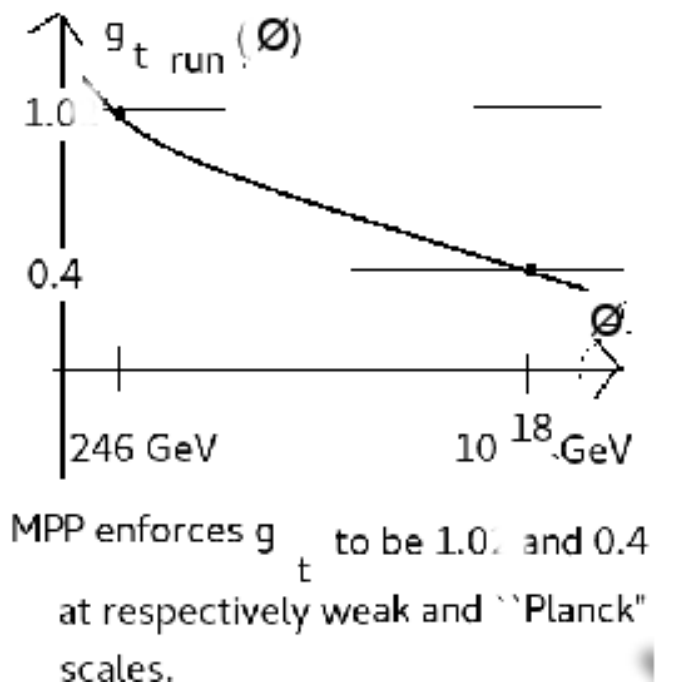

Figure 5: On this figure we see the running top-Yukawa coupling $g_{t \text { run }}(t)$ as a function of the logarithmic scale $t \sim \ln (\phi)$, where the Higgs field $\phi$ is the quantity we have chosen to represent the energy scale $\mu=\phi$. The point is that we have obtained two requirements from the multiple point principle for this running coupling: At essentially the Planck scale $g_{t}=0.4$ and at the scale of the "condensate vacuum", which we presume to be the weak scale, $g_{t}=1.0$.

\section{Scale problem ( hierarchy problem ?)}

- Also in our picture including the "multiple point (criticality) principle", the Higgs mass squared obtains huge quadratically divergent and/or finite terms, when loops are evaluated! 
- But these terms are renormalized to a theoretical requirement of keeping the vacua degenerate.

- So the bare Higgs mass squared is tuned in exactly to cancel the corrections by the "multiple point principle".

- It is not the same as getting rid of them, as would say SUSY, but it has the same effect and makes the getting rid of them superfluous.

\section{Conclusion}

- We have presented a picture/model for

- Dark Matter as 100000 ton, cm big pearls.

- Explanation of the ratio of the weak scale to the "Planck scale".

- Prediction in 1995 of the Higgs mass (in terms of the vacuum expectation value) to be $135 \pm 10 \mathrm{GeV}$. The updated MPP prediction for the Higgs mass is $129.4 \pm 1.8 \mathrm{GeV}$ to be compared with the experimental value $125.38 \pm 0.14 \mathrm{GeV}$.

using only the Standard Model and the "Multiple Point (Criticality) Principle", which only adds information about the parameters of the Standard Model.

- The dark matter model is very successful being able to reproduce the following controversial astronomical observations in addition to the gravitational effects:

- The $3.5 \mathrm{keV} \mathrm{X}$-ray radiation in intensity and frequency.

- The positron emission. Especially the problem that a standard elementary particle type dark matter model tends to give too much continuum spectrum $\gamma$ rays relative to the number of positrons. This problem would be solved in our model, which gives fewer $\gamma$ rays compared to positrons.

\section{Note added in Proof}

After the submission of this paper to the proceedings a referee for another article pointed out that our value $\Delta V=20 \pm 14 \mathrm{MeV}$ was not correct. In fact, including the effect of heavier virtual quarks in the nucleons and the more detailed calculation in e.g. Hai-Yang Cheng, and ChengWei Chiang, "Revisiting Scalar and Pseudoscalar Couplings with Nucleons" arXiv:1202.1292v2 [hep-ph] 18 Jun 2012, we rather get a Higgs nucleon coupling $g_{\phi N N}=1.1 * 10^{-3}$ and thus $\Delta V=$ $1.1 * 10^{-3} * 246 \mathrm{GeV}=270 \mathrm{MeV}$. This is about 13 times larger than the value we used above. Luckily, however, our fits used mainly the combined parameter $\frac{\xi * 10 \mathrm{MeV}}{\Delta V}$ so that only in one (or two) of the quantities we fit, wherein we estimate $\xi$ and $\Delta V$ separately, is it relevant that we have changed the $\Delta V$ estimate. In the other cases we can absorb the change in $\Delta V$ by a compensating change in $\xi=$ the ratio of the actual pearl radius to the critical radius being the smallest one for which the pearls are still stable. Very likely the radius varies from pearl to pearl and the actual radius has to be taken as an average one. If we take, as we must when we consider the $3.5 \mathrm{keV}$ 
radiation, the average weighted with the emission rate of the $3.5 \mathrm{keV}$ radiation, then when the ratio of the times $\frac{t_{\text {spread }}}{t_{\text {radiation }}}$, which is strongly $\xi$ dependent, is below unity the contribution to the $3.5 \mathrm{keV}$ radiation is suppressed. Thus it is very likely that there will be a peaking of the $3.5 \mathrm{keV}$ radiation just where this ratio equals unity. According to our fits above this unity point for the time ratio requires that our fitting parameter $\frac{\xi * 10 \mathrm{MeV}}{\Delta V}=4$ and was described as a lower bound assuming that the ratio is above unity (see Table 2). Using the distribution of pearl sizes weighted with the emission rate of $3.5 \mathrm{keV}$ radiation, we thus get this value as a reasonable prediction.

However the theoretical estimate $\xi=2^{4 / 9} \sqrt{4 \pi}$ used in this paper is for pearl sizes, without any weighting with the radiation intensity, and will thus not be relevant. It is this theoretical radiation un-weighted and therefore not so relevant determination of our parameter $\frac{\xi * 10 \mathrm{MeV}}{\Delta V}$ which we should thus give up including in our fit; it is the only one of our fitting ingredients which actually gets significantly modified by the correction of the value of $\Delta V$. So we should just drop this restriction on the value of our parameter $\frac{\xi * 10 \mathrm{MeV}}{\Delta V}$, given in Table 2 to be 2.4 , which should now be replaced by a value 13 times smaller. But luckily this is not important because it is anyway not expected that the un-weighted pearl size distribution should be used to get e.g. the value of the radiated frequency or the intensity of radiation to be observed from the galactic clusters.

Actually the two experimental quantities fit extremely well to the now most trustable theoretical prediction from the lower bound giving the value $\frac{\xi * 10 \mathrm{MeV}}{\Delta V}=4$ for our parameter: the intensity of the radiation fitted to the radiation from galaxy clusters etc requires the parameter value 3.8 and the $3.5 \mathrm{keV}$ frequency requires the value 5 (see Table 2).

If indeed all the quantities giving rise to the fit - after throwing out "the combined theoretical predictions" - just depended on our chosen parameter $\frac{\xi * 10 \mathrm{MeV}}{\Delta V}$ and not on $\Delta V$ separately, then our fit would only have changed by one of the items having been thrown out. But now we must admit that this is only approximately true: The time ratio, which we put equal to unity for the $3.5 \mathrm{keV}$ radiation weighted distribution of radii, indeed has a relatively weak explicit dependence on $\Delta V$; in fact it behaves as follows

$$
\frac{t_{\text {spread }}}{t_{\text {radiation }}} \propto\left(\frac{\xi * 10 \mathrm{MeV}}{\Delta V}\right)^{5} * \Delta V *(3.55 \mathrm{keV})^{4} .
$$

Note however, that while the $\Delta V$ factor occurring inside our parameter $\frac{\xi * 10 \mathrm{MeV}}{\Delta V}$ comes to the fifth power, the separate occurrence of $\Delta V$ is only raised to the first power. Therefore replacing the separately occurring quantity $\Delta V$ by a 13 times bigger value only increases the time ratio by such a factor 13. Thus the shift in the point on the $\frac{\xi * 10 \mathrm{MeV}}{\Delta V}$ axis by this correction is only by a factor $\sqrt[5]{13}$, which is not so great a shift. In fact the value of our parameter $\frac{\xi * 10 \mathrm{MeV}}{\Delta V}$ corresponding to $\frac{t_{\text {spread }}}{t_{\text {radiation }}}=1$ is shifted from the previous value 4 to $4 / \sqrt[5]{13}=4 / 1.7=2.4$. It is still in the range allowed by the uncertainties.

\section{References}

[1] C.D. Froggatt and H.B. Nielsen, Surveys High Energy Phys. 18, 55-75 (2003); arXiv:hep-ph/0308144.

[2] C.D. Froggatt, H.B. Nielsen, Proc. to the Euroconference on Symmetries Beyond the Standard Model, p.73, (DMFA, Zaloznistvo, 2003); arXiv:hep-ph/0312218. 
[3] C.D. Froggatt, Proc. of 10th International Symposium on Particles, Strings and Cosmology (PASCOS 04), pp.325-334; arXiv:hep-ph/0412337.

[4] D. L. Bennett, C. D. Froggatt and H. B. Nielsen, NBI-HE-94-44, GUTPA-94-09-3, Presented at Conference: C94-07-20 (ICHEP 1994:0557-560), p.0557-560.

[5] D. L. Bennett, C. D. Froggatt and H. B. Nielsen, NBI-95-15, GUTPA-95-04-1, Presented at Conference: C94-09-13 (Adriatic Meeting:0255-279), p.0255-279; arXiv:hep-ph/9504294.

[6] D. L. Bennett and H. B. Nielsen, Int. J. Mod. Phys. A9 5155 (1994).

[7] D. L. Bennett, C. D. Froggatt and H. B. Nielsen, NBI-HE-95-07, Presented at Conference: C94-08-30 (Wendisch-Rietz) p.394-412.

[8] C. D. Froggatt and H. B. Nielsen, Phys. Rev. Lett. 95231301 (2005); arXiv:astro-ph/0508513.

[9] C.D. Froggatt and H.B. Nielsen, Proceedings of Conference C05-07-19.3; arXiv:astro-ph/0512454.

[10] C. D. Froggatt and H. B. Nielsen, Int. J. Mod. Phys. A 30 no.13, 1550066 (2015); arXiv:1403.7177.

[11] C. D. Froggatt and H. B. Nielsen, Mod. Phys. Lett.A30, no.36, 1550195 (2015); arXiv:1503.01089.

[12] C. D. Froggatt and H. B. Nielsen arXiv:2003.05018.

[13] C. D. Froggatt and H. B. Nielsen, Phys. Lett. B368 96 (1996); arXiv:hep-ph/9511371.

[14] H. B. Nielsen and C. D. Froggatt, Presented at Conference C95-09-03.1 (Corfu 1995); arXiv:hep-ph/9607575.

[15] C. D. Froggatt and H. B. Nielsen, PoS CORFU2018, 046; arXiv:1905.00070.

[16] E. Bulbul, M. Markevitch, A. Foster et al., ApJ. 789, 13 (2014); arXiv:1402.2301.

[17] A. Boyarsky, O. Ruchayskiy, D. Iakubovskyi and J. Franse, Phys. Rev. Lett. 113, 251301 (2014); arXiv:1402.4119.

[18] H. B. Nielsen, C. Froggatt, D. Jurman PoS CORFU2017, 075.

[19] N. W. Ashcroft and N. D. Mermin, Solid State Physics Holt, Reinhart and Wilson (2002).

[20] H. A. Jahn and E. Teller, Proc. Roy. Soc. London A161 (1937) 220.

[21] C. D. Froggatt and H. B. Nielsen, Phys. Rev. D80 034033 (2009); arXiv:0811.2089.

[22] H. B. Nielsen, D.L. Bennett, C.R. Das, C.D. Froggatt and L.V. Laperashvili, PoS CORFU2016, 050: arXiv:1705.10749.

[23] J. M. Cline and A. R. Frey, Phys. Rev. D90 123537 (2014); arXiv:1410.7766.

[24] O. Urban, N. Werner, S. W. Allen, A. Simionescu, J. S. Kaastra and L. E. Strigari, MNRAS 451, 2447 (2015); arXiv:1411.0050.

[25] A. Boyarsky, J. Franse, D. Iakubovskyi and O. Ruchayskiy, Phys. Rev. Lett. 115, 161301 (2015); arXiv:1408.2503.

[26] A. Moline, M. A. Sanchez-Conde, S. Palomares-Ruiz, F. Prada MNRAS, 466, 4974 (2017); arXiv:1603.04057.

[27] C. Okoli, J. E. Taylor and N. Afshordi, JCAP 08019 (2018) [arXiv:1711.05271].

[28] N. Hiroshima, S. Ando and T. Ishiyama, Phys. Rev. D97 123002 (2018); arXiv:1411.0050.

[29] J. Navarro, C. Frenk and S. White, ApJ. 462493 (1996). 
[30] A. Burkert, ApJ 447 L25 (1995); arXiv:astro-ph/9504041.

[31] T. Jeltema and S. Profumo, MNRAS 450, 2143 (2015); arXiv:1408.1699.

[32] O.Adriani et al, Nature 458, 607 (2009); arXiv:0810.4995

[33] I.V. Moskaleno and A.W. Strong, Astrophys. J. 493, 694 (1998); arXiv:astro-ph/971012.

[34] R. Adhikari et al, JCAP01, 025 (2017); arXiv:1602.0481.

[35] D.L. Bennett, H.B. Nielsen and I. Picek Phys. Lett. B208, 275 (1988).

[36] C.D. Froggatt and H.B. Nielsen, Origin of Symmetries, World Scientific, Singapore (1991).

[37] A. Kleppe, Proceedings to the $14^{\text {th }}$ Workshop on What Comes beyond the Standard Models, DMFA Zaloznistvo, 103 (2011).

[38] G. Degrassi et al JHEP 08, 098 (2012); arXiv:1205.6497.

[39] CMS Collaboration, Phys. Lett. B805, 135425 (2020), arXiv:2002.06398.

[40] C.D. Froggatt and H.B. Nielsen, Contribution to ICHEP 96, arXiv:hep-ph/9607302.

[41] Gia Dvali, arXiv:1107.0956. 